\title{
Experimental Study on Mechanical Behavior of Perlite Air-Entrained Concrete Material Using DIC Method
}

\author{
Chen Zhiyong \\ Beijing TianJi new materials Co., LTD \\ Beijing, China \\ Chenzy@163.com
}

\author{
Pang Jiazhi \\ School of Aerospace Engineering, Beijing Institute of \\ Technology. Beijing, China \\ pangjiazhi@126.com
}

\begin{abstract}
The basic mechanical properties of the perlite air-entrained concrete (PAC) material were measured using DIC method in this paper, and the deformation behavior and failure mechanism of the material was also researched. The experiment results show that using DIC method could not only exactly measure the elastic modulus and Poisson's ratio but also be efficient in the failure behavior analyzing. It gives another choice when measuring the PAC material and the experiment results also have some reference value for material design and safety applications.
\end{abstract}

Kye words-PAC marerial, digital image correlation, deformation, failure

\section{INTRODUCTION}

Perlite air-entrained concrete (PAC) material [1] is one kind of new building material made from cement and expanded perlite particles with different proportions. PAC material has lots of advantages such as light, high relative strength and thermal insulation, so It has a widely application prospects [2-5] in construction field. In order to give a properly design when using this material, the elastic mechanical parameters (elastic modulus, Poisson's ratio etc.) should be tested first. Meanwhile the deformation behavior and failure mechanism of the material also should be investigated to evaluate its safety performance. But PAC material is a kind of heterogeneous material with lots of expanded perlite particles and holes in it so that the deformation will show heterogeneity characters. These characters should be observed using full field measurement method. Some general experiment method like strain gage is useless when the material has a large non-uniform deformation.

Digital Image Correlation [7-9] (DIC) is an important photomechanical technique widely used for deformation measurements in various fields. By capturing two speckle images from the specimen surface, one before and one after loading (known as the reference and deformed images respectively), DIC is able to resolve the displacement field by comparing and matching image subsets using correlation

\author{
Wang Wenming \\ Beijing TianJi new materials Co., LTD \\ Beijing, China \\ Wangwm@163.com
}

\author{
Ma Qinwei \\ School of Aerospace Engineering, Beijing Institute of \\ Technology. Beijing, China \\ maqw@bit.edu.cn
}

algorithms before obtaining the strain field by numerical differentiation. Because the DIC method could obtain the full field deformation, it is a very suitable technology for large non-uniform deformation observation of PAC material.

The elastic modulus and Poisson's ratio of PAC material are measured using DIC method in this paper and the deformation and failure mechanism of the material are then deeply analyzed and researched. After the introduction to this paper in this section, section 2 outlines the experiments to obtain the elastic parameters and section 3 outlines the results and analysis.

\section{EXPERIMENTS}

The specimen was a cubic (shown in Fig.1) made from PAC material (manufactured by Beijing TianJi new materials Company). The structure of the specimen was designed as the sandwich layered structure that the components ratio in the middle of the specimen was different from the other two sides, the average density is $434 \mathrm{~kg} / \mathrm{m} 3$. The speckle was printed on the surface of the specimen. During the experiment, specimen was compressed with a loading speed of $0.01 \mathrm{~mm} / \mathrm{min}$ and the speckle images were obtained by an Imperx high resolution CCD camera with a speed of $1 \mathrm{fps}$. The experiment setup and the photos are shown in Fig.2.

The deformation results could be obtained by analyzing the speckle images of the specimen using DIC method. The average strain between two points could be calculated through their relative displacement as shown in Fig.3.
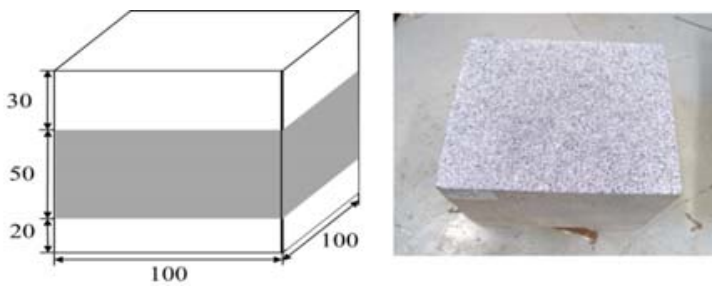

Figure1. The size of the specimen and the photo of specimen with speckle 


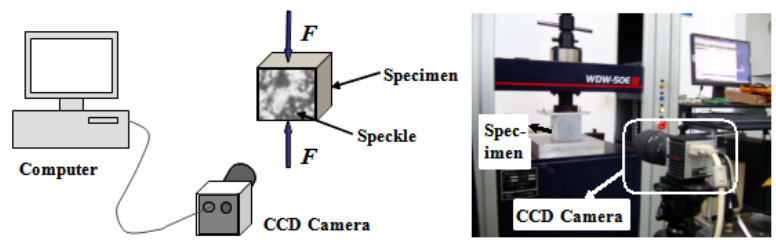

Figure2. The experiment setup and the photos

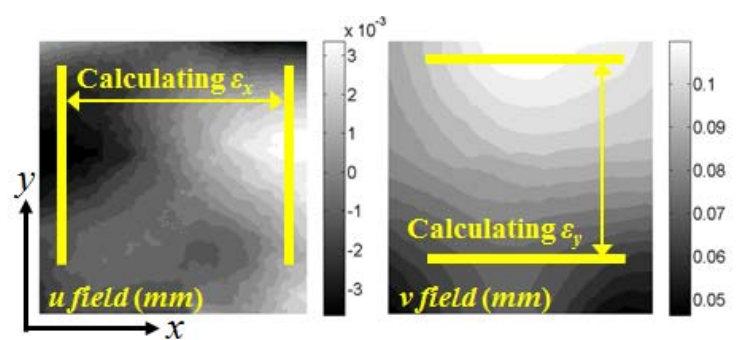

Figure3. The displacement field $(14.5 \mathrm{KN})$ of the specimen calculated using DIC method and the average strain between two points could be calculated through their relative displacement

\section{RESULTS}

The experimental results of load varying with the displacement are shown in Fig.4. Form the curve we can find that the whole loading process could be divided in to three stages: at the beginning of the loading process, the relationship between load and displacement is approximate linear as other homogeneous material; when the load reaches the elastic compression limit (point D in Fig.4), a small decrease appears first and then a rebound happens; after the load exceed the compression limit of the material, the load volatility decline slowly until fully damage of the specimen happens.

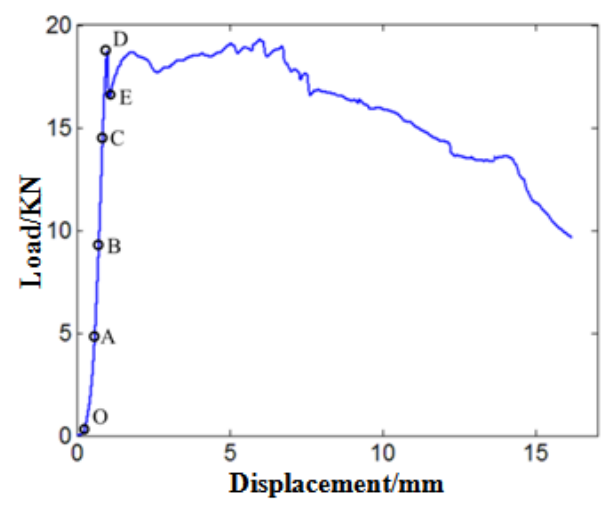

Figure4. The load-displacement curve of the specimen

The region for DIC calculation is marked in Fig.5 and the displacement field under 14.5KN (point C in Fig.4) in both $\mathrm{x}$ and y directions are shown in Fig.3. The stress-strain curve in whole loading process is shown in Fig.6. Then the elastic modulus, Poisson's ratio can be calculated by fitting the linear segments of this curve. There have eight specimen of PAC materials tested in this paper and the maximum load, average sectional area, compressive strength and elastic modulus are listed in Table 1. The consistency of the results shows the repeatability and validity of the experiment. The small difference of parameters between eight specimens may be mainly due to the ratio of the material in the preparation process and the drying time.

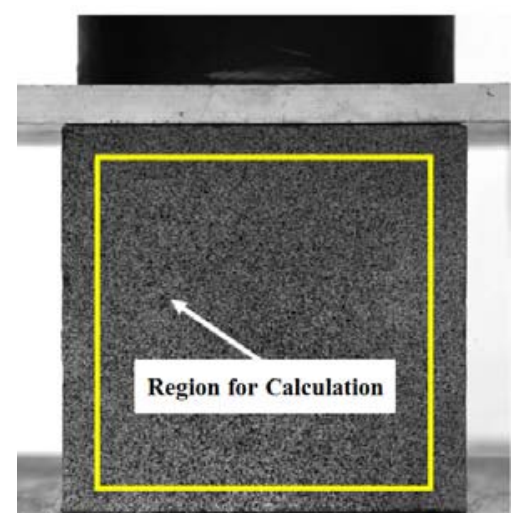

Figure5. The region for DIC calculation

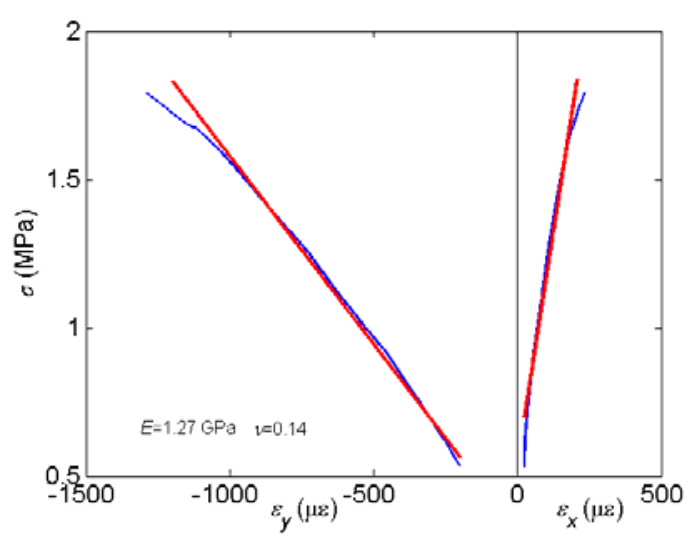

Figure6. The stress-strain curve in both $\mathrm{x}$ and $\mathrm{y}$ directions

TABLE. 1 THE RESULTS OF EIGHT SPECIMEN

\begin{tabular}{ccccc}
\hline No. & $\begin{array}{c}\text { maximum } \\
\text { load (KN) }\end{array}$ & $\begin{array}{c}\text { average } \\
\text { sectional } \\
\text { area } \\
\left(\mathbf{m}^{2}\right)\end{array}$ & $\begin{array}{c}\text { compressive } \\
\text { strength } \\
\text { (MPa) }\end{array}$ & $\begin{array}{c}\text { elastic } \\
\text { modulus } \\
\text { (GPa) }\end{array}$ \\
\hline $1-1$ & 16.65 & 0.010159 & 1.64 & 1.39 \\
$1-2$ & 17.65 & 0.010048 & 1.75 & 1.61 \\
$1-3$ & 17.19 & 0.010070 & 1.70 & 1.49 \\
$1-4$ & 18.83 & 0.010063 & 1.87 & 1.88 \\
$1-5$ & 18.79 & 0.010032 & 1.87 & 1.27 \\
$1-6$ & 18.76 & 0.010140 & 1.85 & 1.96 \\
$1-7$ & 17.83 & 0.010120 & 1.76 & 1.74 \\
$1-8$ & 18.28 & 0.009951 & 1.83 & 1.63 \\
\hline
\end{tabular}

Fig.7 shows the strain fields in y direction under different loading levels (point A, B, C, D, E in Fig.4). They can be used 
to analyze the failure behavior of the specimen. At the beginning of the experiment $(4.8 \mathrm{KN})$, the deformation of the whole specimen was very small and uniform. Then, strain field shown a clear stratification with the loading increase. The deformation at top and bottom was small than that at middle of the specimen. When the load reached the compressive load $(18.6 \mathrm{KN})$, the strain concentration became significant. The strain concentration band appears at the center of the specimen first and then grows along the middle line to the left and right side with the loading increase, finally run through the whole specimen and caused the failure. Furthermore, the position of the real crack shown in Fig.7 is also similar with the strain concentration band so that using the strain field calculated by DIC is proved to be efficient in the failure behavior analyzing.
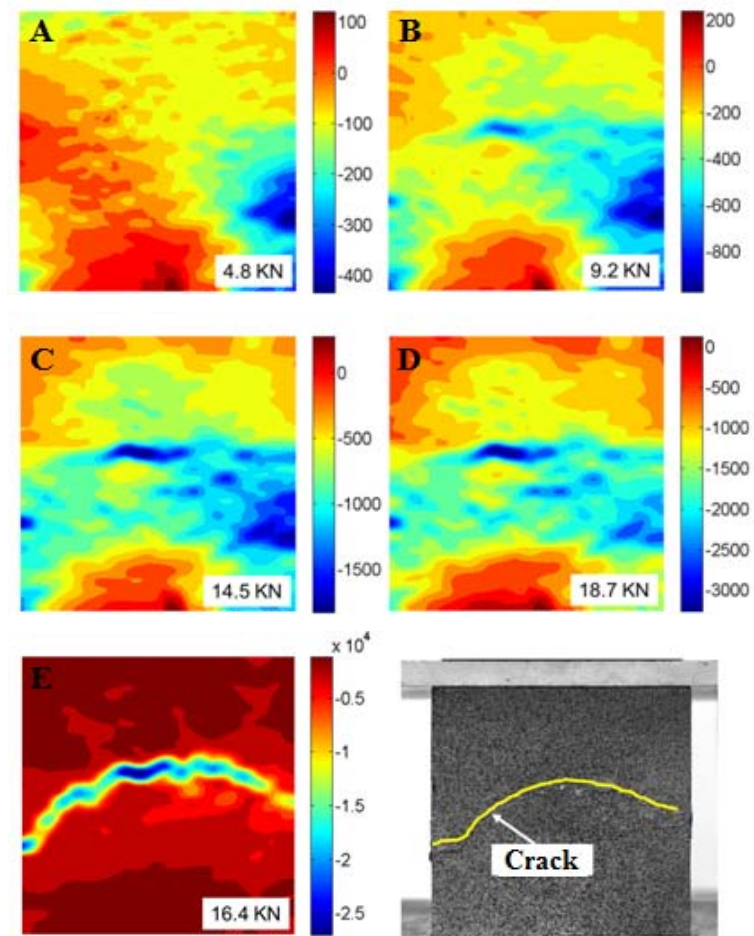

Figure7. the strain fields in $y$ direction under different loading levels

In addition, the micro-observation research shows that the PAC material is a kind of multi-phase composite material. The cement, perlite, crystals in cement block and unhydrated cement particles are mixed together formed the elastic skeleton when deformation happens. But the pores and micro cracks inner the material are considered to be the origin of the damage. From the loading curve, deformation field and the images of the specimen, it can be found that the deformation and failure behavior could be divided into three stages: in stage I, the stress grow from zero to $40 \% \sim 50 \%$ limit compressive strength, the stress concentration born at one point in the material. When the tensile stress at this point exceeds the bonding strength of cement gelatinous and expanded perlite, the micro cracks appear. The crack opening will cause the decrease of stress but the accumulation of residual deformation makes the micro cracks growth and extension; in stage II, the stress reach the ultimate compressive strength, the specimen begin to failure and the crack start unstable expansion; in stage III, the number of cracks increases rapidly and begin expanding and connecting, macroscopic crack appear and the specimen destroy finally.

The phenomenon above shows that the deformation and failure behavior of the PAC material is different to the brittle material like rock and concrete. The damage will not happen immediately when the stress exceed the ultimate compressive strength but hinder the rapid expansion of the crack and materials failure by the material large self- deformation. So the material could absorb much energy in damage, decelerate destruction process and be used as light building materials, fire resisting material, thermal insulation material and acoustic material.

\section{CONCLUSION}

The mechanical properties of PAC material are measured using DIC method in this paper and the deformation and failure mechanism of the material are deeply analyzed and researched through strain field. The experiment results show that the elastic modulus and Poisson's ratio could be exactly measured by DIC method. Meanwhile, the strain fields show the non-uniformity characteristics of deformation and failure modes caused by the sandwich layered structure of the material. The experiment method provided in this paper gives another choice when measuring the PAC material and the experiment results also have some reference value for material design and safety applications.

\section{REFERENCE}

[1] Wang Duolin, "Research on sound absorption properties of expanded perlite material,” Low Temperature Architecture Technology, Vol.33, pp. 13-14, 2011.

[2] Ma Feng, Li Fei and Chen Minghui, "Study on Performance of Palmitic Acid-hexadecanol/expanded Perlite Composit Phase Change Materials," Acta energiae solaris sinica, Vol.31, pp. 1475, 2010.

[3] Liu Yan, Jiang Xiaoshu and Lu Lei, "Research on Preparation and Properties of Capric Acid/Expanded Perlite as Phase Change Material,” Materials Review, Vol.5, pp.315, 2011.

[4] Li Xiaoxue, "Development of Phase Change Energy Storage Building Materials," Science and technology of overseas building materials, Vol. 27, pp.27-29, 2006.

[5] Liu Chao, Ju Fei, Hou Haiyan, et al., "Research Progress of Phase Change Materials for Energy Storage,” Materials Review, Vol.19, pp.261-264, 2005.

[6] Wang Zhiyu, Lin Xutian, Chen Feng, et al, "Preparation of phase change energy storage building material and its performance evaluation,” New Building Materials, Vol.11, pp. 35-37, 2006.

[7] Peters W H, Ranson W. F, "Digital Imaging Techniques in Experimental Stress Analysis,” Opt. Eng, Vol.21, pp.427-431, 1981.

[8] Sutton M A, Cheng M, McNeil S R, et al., “Application of an Optimized Digital Correlation Method to Planar Deformation Analysis,” Comput Vol.4, pp.143-156, 1998.

[9] Bing Pan, Kemao Qian, Huimin Xie, et al., “Two-dimensional digital image correlation for in-plane displacement and strain measurement: a review,” Measurement Science and Technology Vol.20, pp.1-17, 2009.

[10] Jin Guanchang, Meng Libo, Chen Junda, et al., "The Progress and Application of Digital Speckle Correlation Method," Journal of Experimental Mechanics, Vol. 21, pp. 689-702, 2006. 